\title{
The Effects of High-Deductible Health Insurance on Health Trends and Lifestyle
}

\author{
Hrishi P. Joshi \\ Nashua High School South, 36 Riverside St \#1312, Nashua, NH, 03062, U.S.A.; hrishijoshi5@gmail.com
}

\begin{abstract}
Effective health insurance policies are of the utmost importance as they shape the lives of and provide safety to billions of people worldwide. Under the broader umbrella of general health insurance, the high-deductible health plan (HDHP) is a relatively recent idea. The HDHP consists of a high deductible combined with a lower premium. This study strives to find the impact of the HDHP on overall human well-being and health while assessing its effects and identifying potential benefits and drawbacks. The potential benefits of this plan were doubted at first; however, studies have revealed that its popularity has skyrocketed in comparison to its predecessors. HDHP users may experience a series of behavioral consequences of varying effects due to the human psyche. Grouping findings from a personal survey with historical data from other sources and analysis across various demographics demonstrate that HDHP users lead better lives. The representations in the report, implemented with the TI-SmartView TI-84 Plus emulator, illustrate that high-deductible health plans correlate with generally better health outcomes for its users. The survey, which considered wording and response biases, confirms that the above trends are valid irrespective of nominal categorical variables such as gender, education level, and age. This study attests to the fact that the HDHP, when adopted with the understanding of behavioral aspects, can help to improve the health of society as a whole. Finally, it also suggests techniques to make sure that those who select this plan follow certain behaviors that drive positive outcomes.

KEYWORDS: Behavioral and social sciences; Human behavior; Insurance; High-Deductible; HDHP consequences.
\end{abstract}

\section{Introduction}

Uninsured people receive less medical care and they have nowhere to turn to in times of an accident or illness. Essentially, they have worse health outcomes and the benefits of safety most definitely outweigh the costs that must be paid to the insurers. ${ }^{1}$ Whether a person visits the hospital twenty times a year or once a year, medical insurance is equally important to cover the costs of medical expenses related to the patient's risk.

The high-deductible health plan is straightforward: a plan with a high deductible and a lower premium. A deductible is the amount that the insurance holder pays before the insurance company takes over and pays the rest of the medical bills for that person. A premium is the consistent the insurance holder pays based on the plan. This money is paid at certain intervals that could be days, weeks, or years, but is most often months. Maximums for both the premium and the deductible insurance are set for each forthcoming fiscal year by the insurance company.

In addition to the basic details of this plan, another optional clause is connected to this plan versus traditional health plans. Health savings accounts (HSA) are a personal savings account utilized only for healthcare expenses. These medical expenses have a wide variety of ranges in use, from medical to dental and even mental health. The main purpose is to help patients to pay the out-of-pocket costs from their high-deductible plans. They are completely tax-free and any number of people can contribute to these accounts. Shifting the costs to consumers via the high-deductible health plan can help to reduce the overall medical cost. However, as will be shown, the high deductible health plan has several disadvantages as well. ${ }^{2}$

The high-deductible health plan is very popular among people of all ages. Beginning in the year 2004, the high-deductible health plan has quickly grown in use since its inception. From 2007 to 2017, a survey by the "National Health Interview Survey" showed that enrollment in plans of these types increased, whether or not the plan was accompanied by an HSA amongst adults aged 18 to $64 .^{3}$ This same survey shows that no differences in trends is evident amongst different genders. ${ }^{3}$ As a whole, the presence of such plans has increased from $5 \%$ of total plans to $20 \%$ of total plans between the years 2007 and 2013. It is estimated that in the health insurance marketplace, $60 \%$ to $80 \%$ of plans are HDHPs. ${ }^{4}$

At first, HDHP seemed like just a smart business move. However, upon further investigation, this kind of plan can be very detrimental and disadvantageous to certain classes of people. For example, people who are often ill or who have a lower income may be at higher risk of financial bankruptcy. Although it seems that the costs are affordable for people of all levels, the out-of-pocket costs actually have many hidden costs. In addition to the deductible costs, customers still have to worry about copayments and coinsurance. Eventually, people in the two groups stated previously can lose money much more quickly.5 A study by USC confirms this hypothesis. ${ }^{5}$ Those who were part of the high-deductible health group were $10 \%$ more likely to spend $\$ 2,000$ annually on health care in the census and the low-income subgroup. ${ }^{5}$ 


\section{Literature Review}

Clearly, the controversy of high-deductible health insurance is centered around the problems caused by the cost of deductibles. As this is a serious problem, several other studies analyze the influence that low-deductible health insurance has on people of different demographics.

Health spending varies based on factors such as race, age, sex, and much more. Studies show that for all adults, health spending increases as age increases. However, for other nominal variables, many other factors impact how much health spending takes place. Health spending generally refers to the consumer point of view while health expenditure refers to a more general worldly perspective. Thus, it is quite a surprise when the results of Medical Expenditure Panel Survey show that the highest-spending $1 \%$ of the population contributed to more than $20 \%$ of all health expenditures, and that the highest-spending $5 \%$ of the population contributed to more than $50 \%$ of all health spending. In addition to this, the lowest-spending $50 \%$ of the population contributed to only $3 \%$ of total health spending. These figures show the large discrepancies in the "Lorenz curve for medical insurance." This pattern followed for both out-of-pocket spending and overall spending. When this study dives deep into the demographics, many interesting conclusions can be drawn regarding their specific differences. ${ }^{6}$

Factors like age, gender, race, and current health conditions affect the level U.S. citizens spend on insurance. Those above 55 years of age represented more than a quarter of the population of the subjects in the study, however, they accounted for $50 \%$ of total medical spending. Unsurprisingly, the trend followed for people with conditions like heart disease, cancer, and other serious conditions accounted for a higher percentage of total medical spending. ${ }^{6}$ The survey shows that these patterns remain true for high-end populations as well. For people in their 20s up to their 50s, women spend more on insurance than men, however, the difference is mostly negligible. ${ }^{6}$ As for the race, whites dominate almost every single age group in terms of overall health spending. On the other hand, Hispanics and Asians are much more conservative in every age group for all types of health insurance. ${ }^{6}$

A study performed by Sheila R. Reddy et al. ${ }^{7}$ similarly assessed the effects of high deductible health plans using outpatient visits and laboratory/radiology tests. The study was conducted by using a pre-post survey with comparison group study design to find relative decreases in tests and office visits. They eventually discovered a moderate decrease in office visits over the course of the observational study. ${ }^{7}$ Our study differs from this study as we will be using a nonbiased survey to assess the effects that the high-deductible health plan has on the health and lifestyle along with the quantity of patient visits. We will also be comparing the results to notice trends amongst the variables listed in the introduction.

\section{Purpose and Hypothesis}

The last time a major health plan was initiated in the United States was in 2003. George W. Bush and his fellow Republican congressmen experimented when they made a change in the tax code and expressed their thoughts about experimenting with a high-deductible health plan for employers. ${ }^{8}$ The financial crisis later in 2008 caused a boom of these plans within the entire marketplace. However, the last time American democracy truly assessed their insurance needs was nearly 16 years ago. So, what are the problems with this seeming blessing of a plan?

This study aims to provide the above information through the following groups:

A. Find the long-term effects of the high-deductible health insurance plan on subjects of various characteristics. These characteristics include gender, age, education level and years of high-deductible health insurance.

B. Compare these findings with a control group (people who consistently have used low deductible health insurance).

C. Conduct interviews with several medical professionals and perform statistical analysis on the data gathered in the study.

D. Discuss possible solutions (if applicable) based on the findings that match up with the results and help to solve the problem.

It is hypothesized that when a variety of people are subject to surveys regarding their use in high-deductible health insurance when compared to users of low-deductible health insurance, worse health outcomes will be prevalent for holders of HDHP plans when compared to users of LDHP plans.

\section{Results and Discussion}

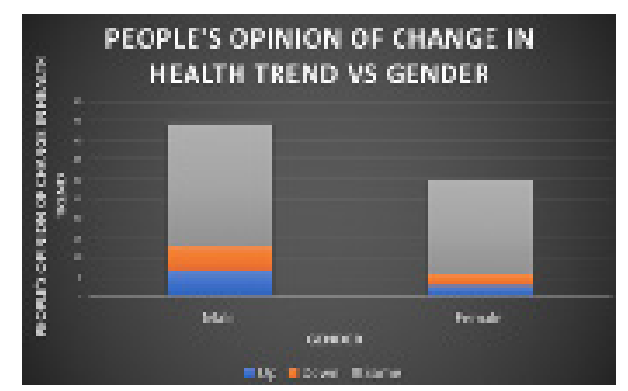

Figure 1. Graph displayed shows how gender effects health trend in subjects. Y־axis is frequency of people's opinion of how their health trend has changed. X-axis is gender Gender does not play a large role in changes in health trends based on insurance type.

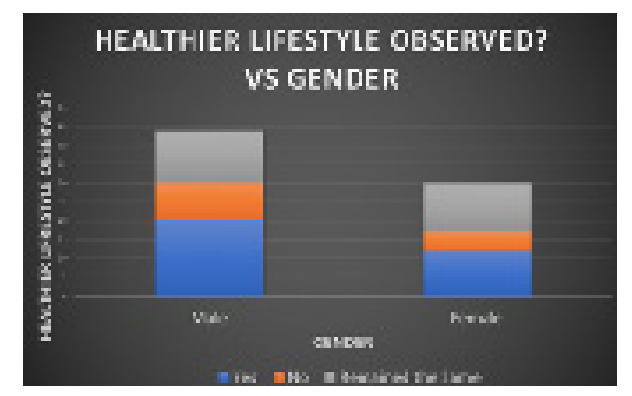

Figure 2. Graph displayed shows how gender affects ability to live a healthy lifestyle in subjects. Y-axis is frequency of each choice for how a subject's lifestyle has change. $\mathrm{X}$-axis is gender. Gender does not play a large role in a lifestyle change based on insurance type. 


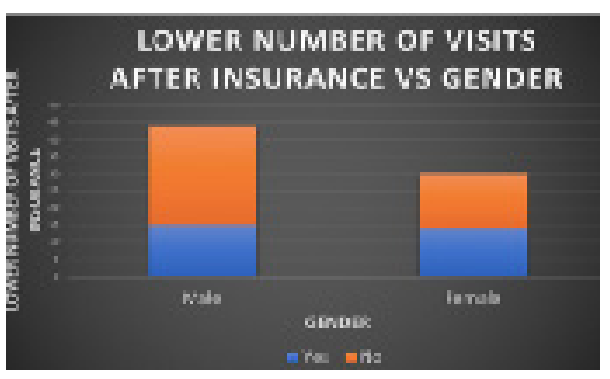

Figure 3. Graph displayed shows how gender affects patient's visits to a doctor. Y-axis is frequency of each choice for "a lower number of visits to the doctor." $\mathrm{X}$-axis is gender. Gender does not play a large role in a change in visit number based on insurance type.

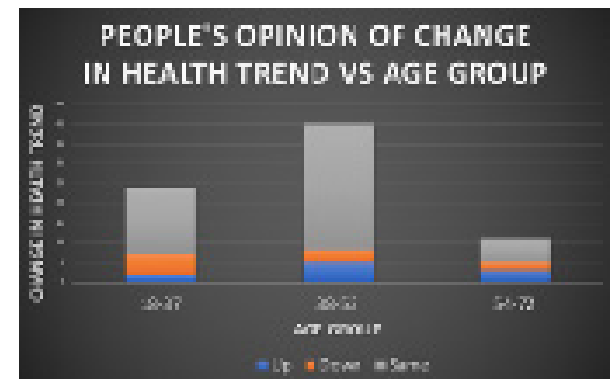

Figure 4. Graph displayed shows how age effects health trend in subjects. Y-axis is frequency of people's opinion of how their health trend has changed $\mathrm{X}$-axis is age Age does not play a large role in health trend changing based on insurance type.

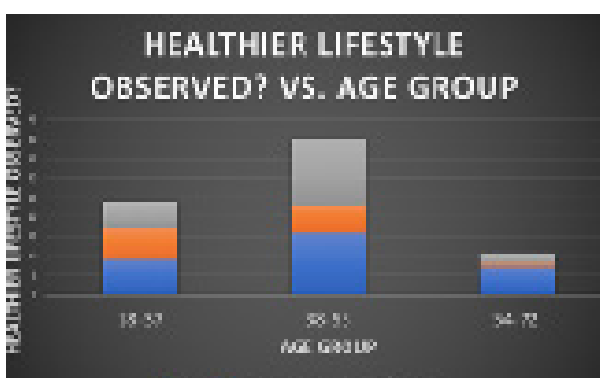

Figure 5. Graph displayed shows how age affects ability to live a healthy lifestyle in subjects. Y-axis is frequency of each choice for how a subject's lifestyle has change. X-axis is age. Age does not play a role in health trend changing based on lifestyle type.

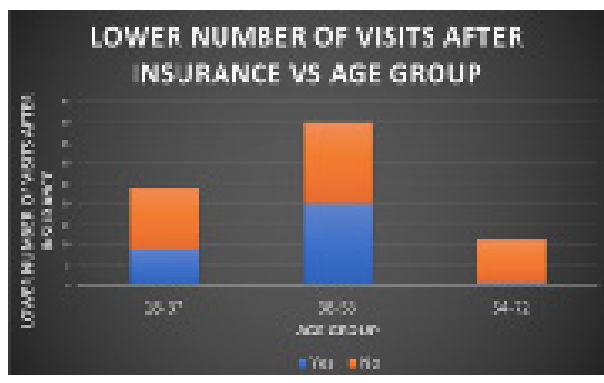

Figure 6. Graph displayed shows how age affects patient's visits to a doctor. Y-axis is frequency of each choice for "a lower number of visits to the doctor." $\mathrm{X}$-axis is age. Age does not play a role in health trend changing based on lifestyle type.

\section{Data Analysis}

The impact of the number of years of high-deductible insurance on high-deductible patients' opinions on their health trend is barely noticeable due to the extreme count of "same" responses (Appendix 1). However, of the responses that had a yes or no answer, Figures 3 and 6 show that the nominal variables of age or gender didn't affect the distribution of responses very strongly. As the appendix later shows, this trend was overarching for all nominal variables tested: age, gender , education, and years of high-deductible health insurance. The graphs also show that after the ability to live a healthier lifestyle after the effects of the insurance was assessed, the distribution of change in lifestyle did not differ much based on either age or gender. However, many more people overall claimed that they lived a healthier lifestyle after this plan. Once again, the appendix shows that for the other nominal variables of education and years of high-deductible insurance, the pattern of negligible response changes follows.

Finally, the last question follows the pattern of negligible responses with respect to nominal variables. Overall, fewer people claimed that they went to the doctor more after possessing high deductible insurance. However, within the nominal variable of age and gender, there was no pattern regarding the number people visiting a doctor. The graphs that associate years of high deductible insurance and education show similar results to those portrayed and explained in the graphs and explanation above since their effect on the three primary questions asked in the survey are negligible as well.

\section{Chi-Square Test \#1: Change in Health Trend}

Null hypothesis: The proportion of "up", "down", and "same" responses is the same across HDHP holders and LDHP holders.

Alternate hypothesis: The proportion of "up", "down", and "same" responses is different across HDHP holders and LDHP holders.

Observed values table (HDHP users):

Table 1 This is the table of observed values for high-deductible health plan users corresponding health trend between the two plans.

\begin{tabular}{|l|l|l|l|}
\hline Response & Up & Down & Same \\
\hline & 10 & 10 & 55 \\
\hline
\end{tabular}

Expected values table (LDHP users):

Table 2. This is the table of observed values for high-deductible health plan users corresponding health trend between the two plans.

\begin{tabular}{|l|l|l|l|} 
Response & Up & Down & Same \\
\hline & 12.80 & 9.15 & 51.22 \\
\hline
\end{tabular}

Chi-square value: 0.97

p-value: 0.62

Since the p-value of 0.62 is above the significance level of 0.05 , I fail to reject the null hypothesis. So, it follows that the proportion of responses is the same across HDHP and LDHP holders.

\section{Chi-Square Test \#2: Lower Number of Visits}

Null hypothesis: The proportion of "yes" and "no" responses is the same across HDHP holders and LDHP holders.

Alternate hypothesis: The proportion of "yes" and "no" responses is different across HDHP holders and LDHP holders.

Observed values table (HDHP users): 
Table 3. This is the table of observed values for high-deductible health plan users corre sponding the decrease in the number of.

\begin{tabular}{|l|l|l|}
\hline Response & Yes & No \\
\hline & 30 & 45 \\
\hline
\end{tabular}

Expected values table (LDHP users):

Table 4. This is the table of expected values for high-deductible health plan users corre sponding the decrease in the number of visits between the two plans.

\begin{tabular}{|l|l|l|}
\hline Response & Yes & No \\
\hline & 21.95 & 53.05 \\
\hline
\end{tabular}

Chi-square value: 4.17

p-value: 0.04

Since the p-value of 0.04 is below the significance level of 0.05 , I reject the null hypothesis. So, it follows that the proportion of responses is the different across HDHP and LDHP holders.

\section{Chi-Square Test \#3: Healthier Lifestyle Observed}

Null hypothesis: The proportion of "yes", "no", and "remained the same" responses is the same across HDHP holders and LDHP holders.

Alternate hypothesis: The proportion of "yes", "no", and "remained the same" responses is different across HDHP holders and LDHP holders.

Observed values table (HDHP users):

Table 5 This is the table of observed values for high-deductible health plan users corresponding the change in lifestyle between people of the two plans.

\begin{tabular}{|l|l|l|l|} 
Response & Yes & No & $\begin{array}{l}\text { Remained the } \\
\text { Same }\end{array}$ \\
\hline & 32 & 16 & 27 \\
\hline
\end{tabular}

Expected values table (LDHP users):

Table 6 This is the table of expected values for high-deductible health plan users corre
sponding the change in lifestyle between people of the two plans.
\begin{tabular}{|l|l|l|l|} 
Response & Yes & $\begin{array}{l}\text { Remained the } \\
\text { Same }\end{array}$ \\
\hline & 38.41 & 12.80 & 23.78 \\
\hline
\end{tabular}

Chi-square value: 2.31

p-value: $0.32 \mathrm{~g}$

Since the p-value of 0.32 is above the significance level of 0.05 , we fail to reject the null hypothesis. So, it follows that the proportion of responses is the same across HDHP and LDHP holders.

As explained in the methods, a chi-square GOF test tests whether the proportion of the subjects is constant throughout the whole population. Since the p-value is below the benchmark p-level of 0.05 , we can reject the null hypothesis for chi-square test \#2. Tables 3 and 4 have a significant difference in their observations. Therefore, there is enough evidence to reject the null hypothesis for the question of whether there is a "lower number of visits to the doctor" after patients switched to the high deductible insurance.
The above data leads to a single major conclusion. The graph that associates lifestyle change with more years of high deductible insurance as the two nominal variables shows that the $\mathrm{HDPH}$ plan results in more people having a perceived similar or better lifestyle rather than a worse lifestyle.

\section{Results and Discussion}

This study effectively identifies the detailed effects of the high-deductible insurance and provides a sensible and realistic approach that combines these results with universal health. The deductibles, copayments, and coinsurance that factor into the decision that is made by potential customers are still large costs that can affect decisions made by customers.

With respect to other studies, this study could be improved in a variety of ways. If more outside data was compared with the data developed from this study, such as more detailed insurance data and hospital data from nearby areas, this study could detect and confirm a stronger correlation between the three main factors under the high-deductible insurance policy. A limitation that comes with this study is the undeniable bias from surveying just a small number of people, allowing for an easy entry of a nonresponse bias and under coverage since everyone who was surveyed did not necessarily give an answer and only a small sample of the population was taken. However, this study could easily be replicated on a larger scale, indicating that a survey where the biases are cautiously recognized and taken care of is a great method of gathering data.

Considering decreasing health after using the HDHP plan is still prevalent despite the high success rates a variety of methods can be used in order to improve the results after the plan. One way is to use a health savings account. Money that is essentially saved from paying less money in the high-deductible account can go towards a health savings account. In a health savings account, money is placed every time money is saved using the high-deductible insurance plan. This idea was initiated so that the health of the users would not be adversely affected. If the money they saved simply ends up going to waste after all the efforts to begin a new plan, the problem would simply arise again. ${ }^{2}$

Doctor visits clearly are an integral part to improve the health of a patient, regardless of the type of insurance. Going to the doctor should not be inhibited due to the extra money that comes out of the patient's pocket. The copayment, the deductibles, and all other costs are simply not worth the risk of great health risks. Since many users responded positively to high-deductible insurances, it is important to remember that money is still an important factor to take full advantage of the plan. When one is dealing with test or procedures that are not extreme or urgent, customers should look for the best financial option that has decent quality to maximize effectiveness and provide reasoning against the low deductible health plan. Another way to maximize effectiveness is to consider online care options offered by specific insurance companies for maintenance and routine issues, such as Teladoc, a company that provides online help for smaller-scale medical problems. 
Some companies, such as Amazon, JPMorgan, and Berkshire Hathaway's joint company Haven Healthcare, are trying to help people who are frustrated with the costs, quality, and service of current health care. ${ }^{9}$ They are attempting to fight rising healthcare costs by attempting to improve patient outcomes, patient experience, and most importantly, lower costs for potential customers. In many ways, the high-deductible health care plan could theoretically result in much lower prices. However, as the introduction showed, despite the high-deductible health care plan clearly having many cost benefits over the low-deductible health plan, people were more likely to spend at least $\$ 2,000$ more annually. Amazon and JPMorgan Chase intend to come out with a plan alongside Haven that completely eliminates the deductible in order to fight this premise. ${ }^{9}$ On the other hand, additional companies are offering only high-deductible health plans to their customers. Despite the high-deductible health plan's quick rise as one of the leading health plans of the $21^{\text {st }}$ century, one must carefully consider the positives and negatives of both LDHPs and HDHPs before making an informed decision about which plan is right for them.

\section{Conclusion}

It was hypothesized that there would be a negative association between high-deductible insurance and the resulting health and lifestyle that these patients endure. Ultimately, this study showed that the high-deductible health insurance plan is correlated to a higher lifestyle change and a decrease in visits to the doctor. These two claims combined show that there is actually a positive correlation between health and increased use of the HDHP plan. Since the survey is just an observational study, no cause-and-effect relationships can be detected; only strong correlations.

Like Reddy's study, the results show that patients overall noticed a decrease in visits to the doctor while observing a healthier lifestyle when comparing LDHP and HDHP users. ${ }^{7}$ Conversely, across all demographics, the studies showed that all demographic groups' health trended at the same level, indicating that the plan was mostly reminiscent of the original low deductible health plan (LDHP). The chi-square test showed that the difference in visits to the doctor was significant, justifying a significant change and providing evidence that the high-deductible insurance plan actually improves their health. The findings about the second and third primary questions, however, paint a different story of the effects of health insurance.

The results from the decrease in visits to the doctor is also shown in Reddy's study. ${ }^{7}$ In Reddy's study, there was a relative 9\% reduction compared to the control group where the subjects who were not in a high-deductible treatment go through. ${ }^{7}$ The graphs show that many more people say "no" to going to the doctors after they have switched to a high-deductible treatment. In addition to the chi-square GOF test developing a conclusion that mirrored Reddy's studies, other nominal variables were tested in both our study and Reddy's study. In both, they were not found to have too much of an impact as it didn't affect how people answered the three primary questions. Although fewer people claimed that they lived a healthier life- style and had a worse health trend, the difference was miniscule and thus statistically insignificant by means of the chi-square test.

Finally, when investigating how specific variables such as age, gender, years of high-deductible insurance, or education affected any of the three primary questions, the graphs once again show that none of the possible nominal variables have a sizable impact on the distribution of any of the answers. These findings mirror the study done by the Center for Disease Control and Prevention. ${ }^{3}$ However, they refute the evidence provided in Kamal's ${ }^{6}$ study, which states that people of different ages do indeed influence the lifestyles and health different, and therefore the spending habits of these groups.

An unsupported conclusion without in-depth research would be that high-deductible plans are disastrous due to certain characteristics that entail the plan. However, the survey responses clearly suggest that people think they alter their lifestyle when they sign-up for this plan. The plan has a substantial financial benefit for healthy individuals. People realize this and actually change to healthier life style which reduces the need for doctor visits and at the same time improve health creating a virtuous cycle.

\section{Methods}

In order to gather information about high-deductible insurance tendencies, the intention was to survey a variety of individuals with differing ethnicities, age, and gender. This data was then compared with data from insurance companies and hospitals corroborating with the author's personal research. This information was then analyzed using different methodologies. These methodologies, which will be explained in depth later, include a chi-square test to find differences between a control group and an experimental group and general graph analysis techniques.

These were the questions asked in the survey in December of 2019. The survey was given as a mail survey on Google Forms and 117 people were surveyed. The subjects were chosen through social media groups. The questions were determined based on what questions could be used to create data that would accurately portray the effects of the high-deductible health plan on lifestyles:

1. What is your age group? (Options: $18-37,38-53,54-72$ )

2. What is your gender? (Options: Male, Female)

3. What is your ethnicity? (Options: Asian American, White American, Black or African American, Hispanic or Latino, Native American and Alaska Native, and Native Hawaiian and Other Pacific Islander)

4. What is your highest level of education? (Options: Didn't Graduate High School, High School Diploma, Associates, Bachelors, Masters, Doctorate)

5. Have you been on a high deductible insurance plan? (Options: Yes, No)

6. How long have you been on the plan? (No options)

7. Does the fear of copayment and deductibles keep you from going to the doctor when you would have otherwise? (Options: Yes, No) 
8. Has your health level trend been up, down or about the same? (Options: Same, Up, Down)

9. Have you changed your lifestyle to healthier because of the high cost of healthcare? (Options: Yes, No, Remained the Same)

As part of the analysis, a chi-square test was used to analyze the collected data. Specifically, we used a chi-square GOF test. ${ }^{10}$ In this test, a set of numbers in a statistical chart is used to define a data set that are the observed values for an experimental data set. For example, if the overall health trend increase was investigated and found to be positive among people with high-deductible insurance, the chi-square test will confirm that the null hypothesis is either true or false. In the Chi-Square goodness of fit test, the null hypothesis assumes that there is no significant difference between the observed and the expected value. The alternate hypothesis assumes that there is a significant difference. The chi-square value then creates a $\mathrm{p}$-value. If this $\mathrm{p}$-value is below a certain threshold, the null hypothesis is rejected and the alternate hypothesis is accepted.

\section{Chi-Square Test}

The following model is taken from the TI-SmartView TI84 Plus emulator.

Note: The below values are completely unrelated to the subject of insurance and this research.

First, a set of numbers are listed as the "observed counts" (column 1) along with a set of numbers that are labeled as the "expected counts" (column 2).

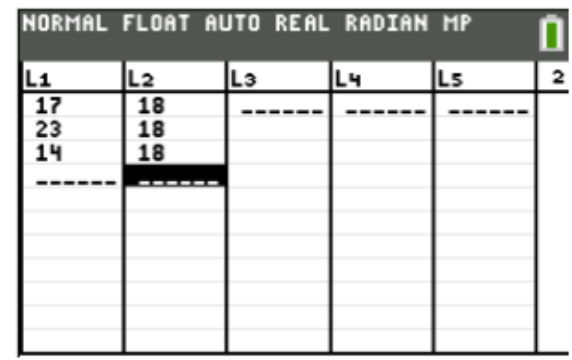

Figure 7. This calculator screenshot represents the sample data.

Then, the chi-square value and the corresponding $\mathrm{p}$-value are created.

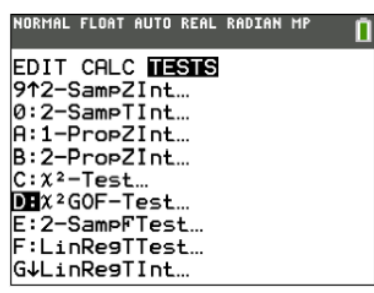

Figure 8. This calculator screenshot shows where to go to actually find the chi-square test to use.

In the case of this study, the threshold p-value was 0.05 .
How exactly are the calculations made for the chi-square $\mathrm{GOF}$ test?

$$
x_{c}^{2}=\sum \frac{\left(O_{i}-E_{i}\right)^{2}}{E_{i}}
$$

Formula 1 This is the chi-square GOF test formula. Oi is the observed value of every data point while $\mathrm{Ei}$ is the expected value of every data point.

This value is the chi-square value that determines if the expected proportions for each aspect of the nominal variable matches up with the theoretical model.

What exactly does this chi-square value explain? The chisquare value dictates the $\mathrm{p}$-value. If the $\mathrm{p}$-value is less than 0.05 , then there is considered a significantly large difference between the expected and the observed values.

We conducted three major chi-square tests. They were not associated with the specific nominal variables; instead, they associated all participants in the survey who stated that they were on a high-deductible plan with three major categories (Change in Health Trend, a Lower Number of Visits, and Healthier Lifestyle Observed). The observed values were determined by looking at the low-deductible insurance members surveyed and assessing their responses since they were the "control group" in this case. This proportion was then applied to the total number of people in the observed group to find what the expected numbers that should have been? In order for the test conducted to be fair. Since the number of people surveyed from each group (types of insurance) were different, using just the raw numbers for each response value (e.g. "yes”, "no", "same”) would deter the results.

The primary biases that could have affected the surveying of the participants in the survey were nonresponse bias and response/wording bias. Through a variety of ways, the effects of these biases were limited so the results would accurately reflect the true stance of the population. Response bias is a type of bias where the question is worded in such a way that the responder changes their response to give a different, or more socially acceptable, answer. The list of questions used in my survey explicitly avoided the wording of "due to insurance" when assessing how the effects of not going to the doctor or lowering the overall lifestyle/health of the insurance users. In addition, a method through which response bias was decreased was through by not asking any personal questions regarding the medications that certain people are taking or the diseases that some people have.

The nonresponse bias, specifically, is a form of bias where the response rate of a particular survey or questionnaire is very low compared to other forms of collecting data. This is an especially common case in mail surveys, such as the one used in this survey. Due to this bias having the ability to easily skew the data, a variety of techniques were undertaken to limit the effects of this bias as much as possible. For one, the survey was kept short ( 9 questions that were mostly multiple choice) so that people would not have to take too much time to answer the survey questions. Secondly, all subjects were aware that the information was anonymous and even their names would not 
be recorded. Finally, reminders were sent on an incremental basis for the audience to respond. All of these combined resulted in a respectable and unbiased survey to use as data in this research paper.

\section{Appendix}

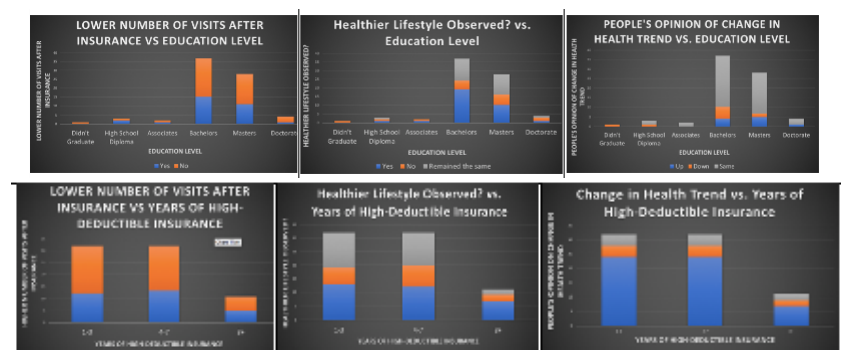

Note- The graphs showing the trends of ethnicity were highly biased as not all ethnic groups were properly represented in the census of my survey. Therefore, charts and graphs relating to this are not included in this research paper.

\section{Acknowledgements}

This research project was made possible through the support of my family, my friends, my teachers, and my mentors. I would like to show my appreciation to the following influential contributors for their unequivocal support: First, I would like to thank Mr. Ketan Kumar from Massachusetts Institute of Technology for carefully reviewing my papers and thereafter offering expert advice to improve upon my drafts. Next, I would like to thank Miss Mary Zhu from Stanford University for critical and vital advice to help me improve and polish my papers to the highest level. Finally, I would then like to thank my parents, Chakshu and Paresh Joshi, for their unwavering trust, confidence, and support in all of my ventures.

\section{References}

1. Bovbjerg, Randall R.; Jack Hadley. Urban Institute. www.urban.org/research/publication/why-health-insurance-important (accessed June 17, 2020).

2. Folger,J.Investopedia.

www.investopedia.com/articles/personal-finance/090814/pros-andcons-health-savings-account-hsa.asp (accessed June 17, 2020).

3. Cohen, R. A.; Zammitti E. P. High-deductible Health Plan Enrollment Among Adults Aged 18-64 With Employment-based Insurance Coverage, 2018. Centers for Disease Control and Prevention. www.cdc.gov/nchs/products/databriefs/db317.html (accessed June 17, 2020).

4. Appold, K. Managed Healthcare Executive. www.managedhealthcareexecutive.com/mhe-articles/highdeductible-health-plans-brief-history (accessed June 17, 2020).

5. Gersema, E. High-Deductible Health Plans Raise Risk of Financial Ruin for Vulnerable Americans, Study Finds, 2018. University of Southern California.

news.usc.edu/140182/high-deductible-health-plans-raise-riskof-financial-ruin-for-vulnerable-americans-study-finds/ (accessed 17 Jun 2020).

6. Kamal, R. Peterson-Kaiser Health System Tracker. www.healthsystemtracker.org/brief/how-health-spending-patternsvary-by-demographics-in-the-u-s/ (accessed 17 Jun 2020).

7. Reddy, S. R.; Ross-Degnan, D.; Zaslavsky, A. M.; Soumerai, S. B.; Wharam J. F. Impact of a high-deductible health plan on outpatient visits and associated diagnostic tests.

Med Care [Online] 2014, 52(1), 86-92.

https://www.ncbi.nlm.nih.gov/pmc/articles/PMC5147026/.
8. Haefner, M. Becker's Hospital Review.

https://www.beckershospitalreview.com/payer-issues/analysis-highdeductible-health-plans-broke-the-us-health-insurance-system.html (accessed 17 Jun 2020).

9. Thorne, J. GeekWire.

www.geekwire.com/2019/amazon-jpmorgan-roll-new-healthinsurance-plans-part-haven-joint-venture/ (accessed 17 Jun 2020).

10. StatTrek.

https://stattrek.com/chi-square-test/goodness-of-fit.aspx (accessed 17 Jun 2020)

\section{Author}

Hrishi Joshi is a sophomore attending Nashua High School South. He is passionate about stem subject. He has taken several AP courses at an early age such as AP Computer Science and $\mathrm{AP}$ Calculus $\mathrm{BC}$ and hopes to pursue a career in computer science. 